\title{
Concise update on colorectal cancer epidemiology
}

\author{
Camilla Mattiuzzi $^{1}$, Fabian Sanchis-Gomar ${ }^{2}$, Giuseppe Lippi $^{3}$ \\ ${ }^{1}$ Service of Clinical Governance, Provincial Agency for Social and Sanitary Services, Trento, Italy; ${ }^{2}$ Department of Physiology, Faculty of Medicine, \\ University of Valencia and INCLIVA Biomedical Research Institute, Valencia, Spain; ${ }^{3}$ Section of Clinical Biochemistry, University of Verona, \\ Verona, Italy \\ Contributions: (I) Conception and design: G Lippi; (II) Administrative support: None; (III) Provision of study materials or patients: G Lippi, C \\ Mattiuzzi; (IV) Collection and assembly of data: All authors; (V) Data analysis and interpretation: All authors; (VI) Manuscript writing: All authors; \\ (VII) Final approval of manuscript: All authors. \\ Correspondence to: Prof. Giuseppe Lippi. Section of Clinical Biochemistry, University Hospital of Verona, Piazzale LA Scuro, 37134 Verona, Italy. \\ Email: giuseppe.lippi@univr.it.
}

\begin{abstract}
Colorectal cancer is a type of gastrointestinal malignancy originating from either the colon or rectum. In this short report we provide a concise update on recent colorectal cancer statistics, especially concerning frequency, mortality, life expectancy and risk factors. Overall, colorectal cancer is the third more frequent malignant disease around the world (1.85 million of new cases/years; $10.2 \%$ of total malignancies), with $2.27 \%$ cumulative risk of onset between $0-74$ years. The age-standardized rate increases by over 10 -fold before the age of 50 up to $\geq 85$ years, whilst men have $~ 50 \%$ enhanced risk compared to women (the $0-74$ years risk is $2.75 \%$ in men and $1.83 \%$ in women, respectively). Although colorectal cancer screening has contributed to slightly reduce the number of diagnoses at advanced stages, most cases are only identified after symptoms onset. The number of worldwide deaths for colorectal cancer has been estimated at 0.88 million in 2018 , representing $\sim 1.4 \%$ of all-cause and $\sim 8.9 \%$ of cancer-related deaths, with over $30 \%$ increase occurred during the past 15 years and a further $25 \%$ growth expected by the year 2030. The cumulative risk of dying for colorectal cancer is $0.92 \%$ between $0-74$ years ( $1.14 \%$ in men and $0.72 \%$ in women, respectively). The 5 -year cumulative survival is $64-67 \%$, being $89-90 \%$ in patients with localized cancer, $70-71 \%$ in those with regional cancer, decreasing to $14-15 \%$ in those with distant cancer. The leading risk factors include familial history, pre-cancerous conditions, tall stature, physical inactivity, overweight, large intake of alcoholic beverages, high consumption of red or processed meat, as well as modest intake of dairy products and foods containing wholegrains or dietary fibre. The association between colorectal cancer and human development index suggests that reinforcement or timely establishment of preventive measures and accurate screening programs may be advisable, especially in countries undergoing considerable societal and economic changes.
\end{abstract}

Keywords: Colorectal cancer; epidemiology; statistics; frequency; mortality

Submitted Jul 05, 2019. Accepted for publication Jul 12, 2019.

doi: 10.21037/atm.2019.07.91

View this article at: http://dx.doi.org/10.21037/atm.2019.07.91

\section{Introduction}

Colorectal cancer is a type of gastrointestinal malignancy originating from either the colon or the rectum. Although both forms can be simply defined as colon or rectal cancers, depending on their origin, they are often merged because of the many biologically and clinically common features (1). Adenocarcinoma is the most frequent colorectal malignancy (up to $95 \%$ of cases), followed by carcinoid tumors, gastrointestinal stromal tumors (GISTs), lymphomas and sarcomas (2). Well differentiated adenocarcinoma presents with over $95 \%$ of tumor gland formation, whereas gland formation is only $50-95 \%$ or $<50 \%$ in moderately and poorly differentiated adenocarcinomas, respectively. Moderately differentiated adenocarcinoma is the most frequent diagnosis $(\sim 70 \%)$ in clinical practice, whilst poorly 
or well differentiated adenocarcinomas account for $20 \%$ and $10 \%$ of cases, respectively (2). The left rather than the right colon is more frequently affected (i.e., 5:1).

Colorectal cancer may be unluckily silent for long in a large number of patients, at least until it substantially grows and spreads, thus adversely impacting the prognosis. In symptomatic patients, the disease may generate changes in bowel motility (e.g., diarrhea or constipation), constant sensation of needing bowel movements, occult or evident colorectal bleeding, as well as abdominal discomfort, cramping, unexplainable weight loss, weakness and fatigue, especially in patients with advanced stage cancers (1). In the following part of this short report we provide a concise update on recent colorectal cancer statistics, especially concerning frequency, mortality, life expectancy and risk factors.

\section{Frequency}

According to the last statistics of the International Agency for Research on Cancer (IARC) of the World Health Association (WHO) (3), colorectal cancer is the third more frequent malignant disease around the world (1.85 million of new cases/year; $10.2 \%$ of total malignancies), only preceded by lung (2.09 million of new cases/year; $11.6 \%$ of total malignancies) and breast (2.08 million of new cases/year; $11.6 \%$ of total malignancies) cancers. Colorectal cancer is also the third most common type of malignancy in men (1.03 million of new cases/year), after lung and prostate cancers, and the second most frequent malignancy in women $(0.82$ million of new cases/year), after breast cancer. Provided that current predictions are reliable, the global burden of colorectal cancer is expected to approximate 2.2 million new cases per year in 2030, thus exhibiting a further $20 \%$ increase (4).

The incidence of colorectal cancer is higher in Australia and New Zealand (36.7 cases per 100,000), followed by Europe (28.8-32.1 cases per 100,000), Eastern Asia (26.5 cases per 100,000) and North America (26.2 cases per $100,000)$, whilst is the lowest in Africa (6.4-9.2 cases per $100,000)$ and South-Central Asia (4.9 cases per 100,000). The worldwide cumulative risk of developing colorectal cancer is $2.27 \%$ between $0-74$ years $(2.75 \%$ in men and $1.83 \%$ in women), higher in countries with very high human development index (HDI; $3.56 \%$ between $0-74$ years) than in those with low HDI $(0.81 \%$ between $0-74$ years). Interestingly, Hungary is the country with the highest agestandardized rate $(51.2$ cases per 100,000$)$, followed by South
Korea (44.5 cases per 100,000$)$, Slovakia (43.8 cases per $100,000)$ and Norway $(42.9$ cases per 100,000) (3). Although the frequency remains higher in highly developed countries, the trend has recently stabilized or even decreased, whilst an increased incidence has been recently observed in some low- and middle-income countries, perhaps reflecting the adoption of more western lifestyles (4). Recent evidence attests that colon cancer recurrence can be as high as $17 \%$, and less than half of such cases are diagnosed during nonscheduled interval visits (5).

The risk of colorectal cancer increases with ageing, becoming more common in subjects aged 50 years of older. The median age at diagnosis is 72 in women and 68 in men, respectively. Notably, the number of colon cancer cases is $1.5-5.4$ per 100,000 between $25-39$ years (1.4-5.3 in men and 1.6-5.5 in women), increases to $10.3-18.5$ per 100,000 between 40-49 years (10.2-18.9 in men and 10.4-18.1 in women), 34.3-62.5 per 100,000 between 50-64 years (37.4-72.7 in men and 31.2-53.2 in women), 92.6-212.2 per 100,000 between 65-85 years (107.7-229.7 in men and $79.2-200.4$ in women), up to 240.9 in subjects aged 85 years or older (264.0 in men and 229.2 in women), thus exhibiting an over 10-fold increase before the age of 50 to $>85$ years (1).

As specifically concerns colon cancer, 1.097 million cases have been diagnosed in 2018 ( 0.576 million in men and 0.521 in women), accounting for a global cumulative risk of $1.31 \%$ between $0-74$ years (1.51\% in men and $1.12 \%$ in women). The cumulative risk of developing colon cancer is considerably higher in Australia and New Zealand (2.59\% between $0-74$ years), Europe (2.00-2.41\% between $0-74$ years) and North America (1.82\% between 0-74 years) compared to Africa (0.30-0.96\% between 0-74 years) and South-Central Asia (0.29\% between $0-74$ years), as well as in countries with very HDI (2.18\% between $0-74$ years) compared to those with low HDI ( $0.40 \%$ between $0-74$ years) (3). A total number of 0.704 million rectal cancer cases have been diagnosed in 2018 ( 0.430 million in men and 0.274 in women), accounting for a global cumulative risk of $0.91 \%$ between $0-74$ years $(1.20 \%$ in men and $0.65 \%$ in women). The cumulative risk of developing rectal cancer is considerably higher in Europe $(1.17-1.55 \%$ between $0-74$ years), Australia and New Zealand (1.40\% between $0-74$ years) and Eastern Asia (1.35\% between $0-74$ years) compared to Africa $(0.27-0.52 \%$ between $0-74$ years) and South-Central Asia ( $0.24 \%$ between $0-74$ years), as well as in countries with very high HDI (1.30\% between $0-74$ years) compared to those with low HDI $(0.32 \%$ between 0-74 years) (3). 


\section{Mortality data}

Malignant diseases are the second leading cause of mortality around the world, accounting for approximately 9.6 million deaths in 2018. According to the last update of the WHO mortality database (6), the number of worldwide deaths for colorectal cancer has been estimated at approximately 0.794 million in 2016, thus representing $1.40 \%$ of all deaths and $8.86 \%$ of cancer-related deaths, respectively. The same figures in the year 2000 were approximately 0.595 million, $1.14 \%$ and $8.48 \%$, thus underlining that a remarkable increase has occurred in the past 15 years, in terms of overall number of deaths ( +0.199 million, i.e., $+33 \%)$, as well as of all-cause $(+23 \%)$ and colorectal cancer-related $(+4 \%)$ mortality. A provisional update for the year 2018 would raise the number of worldwide colorectal cancer deaths up to approximately 0.88 million, with a further $\sim 10 \%$ increase from 2016 (3). Provided that the current trend cannot be reversed, colorectal cancer mortality is expected to approximate 1.1 million deaths per year in 2030, thus exhibiting a further $25 \%$ increase (4). Notably, colorectal cancer has progressed from the $20^{\text {th }}$ to the $17^{\text {th }}$ place among the first 20 causes of worldwide mortality from 2000 to 2016 (ischemic heart disease, stroke and chronic obstructive pulmonary disease are still the leading causes, with 9.4, 5.8 and 3.0 million deaths per year). Among the more frequent causes of death for malignant diseases, colorectal cancer is only preceded by trachea, bronchus or lung (1.7 million deaths per year) and liver ( 0.83 million deaths per year) cancers. Regarding specific geographical areas, colorectal cancer is the $20^{\text {th }}$ cause of death in the South-East Asia region, but is ranked $7^{\text {th }}$ in the European region and $11^{\text {th }}$ in the American region. The cumulative risk of dying for colorectal cancer is $0.92 \%$ between $0-74$ years $(1.14 \%$ in men and $0.72 \%$ in women) (3), being substantially higher in Europe (1.04-1.80\% between $0-74$ years) than in Africa ( $0.52-0.74 \%$ between $0-74$ years), as well as in countries with very high HDI $(1.16 \%$ between 0-74 years) compared to those with low-medium HDI (0.51-0.60\% between $0-74$ years). The latest WHO global estimates for the year 2016 also attest that colorectal cancer causes 19.1 million cause-specific disability-adjusted life year (DALYs) and 18.5 million years of life lost (YLLs), both exhibiting a $30 \%$ increase during the past 15 years (6).

\section{Prognosis}

The 5-year survival rate approximates $90 \%$ when colorectal cancer is diagnosed at an early stage, compared with $13 \%$ when the diagnosis is delayed (3), which is mostly due to the lengthy silent nature of disease and the still limited number of tests that can be used for timely and efficient screening or diagnosis (7-9). Official statistics on prognosis of colorectal cancer have been issued by the American Cancer Society (AMS) (1). Overall, the 5-year cumulative survival of patients with colon cancer is $64 \%$, with $90 \%$ survival in those with localized cancer (e.g., stages I, IIA, and IIB), $71 \%$ in those with regional cancer (stages IIC and III), but decreasing to $14 \%$ in those with distant cancer (stage IV). As regards rectal cancer, the 5-year cumulative prognosis is quite similar (i.e., 67\%), with $89 \%$ survival in patients with localized cancer (e.g., stages I, IIA, and IIB), $70 \%$ in those with regional cancer (stages IIC and III), also decreasing to $15 \%$ in those with distant cancer (stage IV). Although reinforced efforts made on colorectal cancer screening have contributed to slightly reduce the number of diagnoses at advanced stages, most cases-up to $90 \%$ are still diagnosed after symptoms onset, by diagnostic colonoscopy $(\sim 80 \%)$ or even during emergent surgery $(\sim 10 \%)(10)$. Importantly, a recent survey on the impact of screening policies based on fecal occult-blood testing (FOBT) has revealed that this test has been successful in lowering colorectal cancer-related deaths by $20-30 \%$, but seems otherwise less efficient to substantially improve allcause mortality (11), thus calling for identification and establishment of more effective screening strategies.

\section{Risk factors}

The leading risk factors of colorectal cancer, as recently emphasized by the IARC, are summarized in Table 1, along with the relative level of evidence and strength $(1,3)$. Most of these are dietary behaviors, whilst obesity and physical inactivity are now also considered important risk factors (12). Additional conditions predisposing to colorectal cancer include inflammatory bowel disease (e.g., ulcerative colitis or Crohn's disease), cigarette smoke, whilst between $5-10 \%$ cases originate from hereditary diseases (e.g., pre-cancerous conditions) such as familial adenomatous polyposis and Lynch syndrome (also known as hereditary nonpolyposis colorectal cancer) or, less commonly, are associated with Peutz-Jeghers syndrome (PJS) and $M Y H$-associated polyposis (MAP). Nearly $20 \%$ of people who develop colorectal cancer have familiarity for these conditions, especially when the disease has been diagnosed before the age of 45 and in more than one first-degree relative $(1,12)$. 
Table 1 Leading risk factors of colorectal cancer

\begin{tabular}{lcc}
\hline Risk factor & Level of evidence & Increased risk (fold) \\
\hline Tall stature & Strong & $1.07(\mathrm{per} 6.5 \mathrm{~cm})$ \\
Familiarity & Strong & $2.0-4.0$ \\
Pre-cancerous conditions & Strong & 1.7 \\
Physical inactivity & Strong & $1.3\left(\mathrm{BMI} \geq 30 \mathrm{~kg} / \mathrm{m}^{2}\right)$ \\
Overweight and obesity & Strong & $1.2(>100 \mathrm{or}>50 \mathrm{~g} / \mathrm{day})$ \\
Large intake of red or processed meat & Strong & $1.1-1.2$ \\
Modest intake of foods containing wholegrains or dietary fibre & Strong & 1.2 \\
Diet poor of dairy products & Strong \\
Consumption of alcoholic beverages & Strong & $1.4(>3 \mathrm{drinks})$ \\
Poor consumption of fish & Limited & $\mathrm{NA}$ \\
Vitamin C and D deficiencies & Limited & $\mathrm{NA}$ \\
\hline
\end{tabular}

BMI, body mass index; NA, non-available.

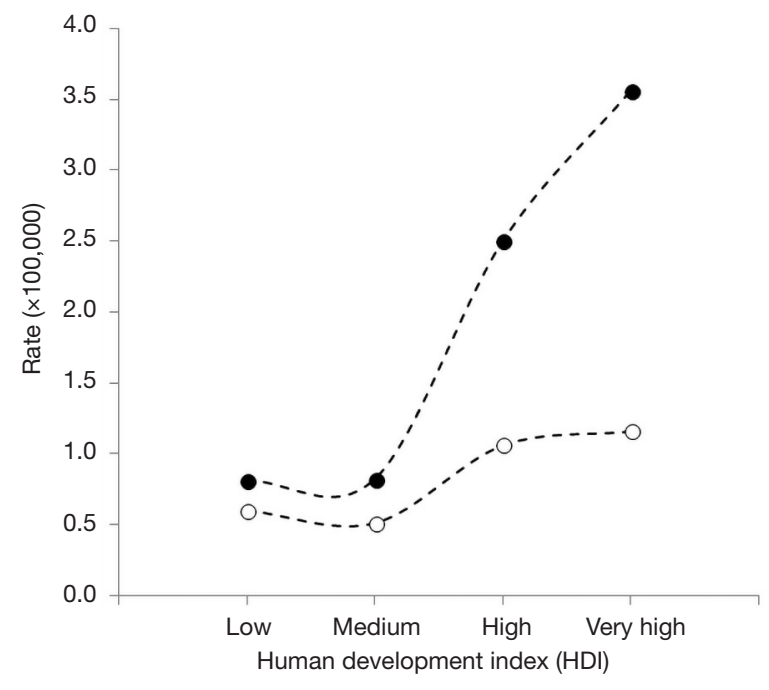

Figure 1 Relationship between frequency $(\bullet)$ and mortality $(\circ)$ rates of colorectal cancer and human development index (HDI).

Patients with polyps $>1 \mathrm{~cm}$, with $>2$ polyps or displaying dysplasia have a higher risk of developing colorectal cancer. Enhanced risk has also been reported in subjects with type 2 diabetes, but it is not clear whether this link is genetic of simply environmental (i.e., due to the impact of joint risk factors, such as physical inactivity and obesity) (1). Reliable evidence garnered on over 2.1 million subjects shows that colorectal cancer mortality can be reduced by $20 \%$ with high adherence to the Mediterranean diet (13). Another recent meta-analysis concluded that red and processed meat, saturated or animal fats and cholesterol, preserved and spicy foods are associated with enhanced risk of colorectal cancer in Asia, whilst calcium, dairy aliments, vegetables and fruit as well as selenium, lycophene, carotenoids, may have a protective role against (14).

\section{Conclusions}

This concise overview of recent colorectal cancer statistics provides a fertile ground for addressing present and future healthcare interventions aimed at preventing, diagnosing and managing this prevalent and severe disease. The frequency and mortality remain both troublingly high, since this malignancy is a paradigmatic example of disease strongly influenced by lifestyle, nutrition and environment. Recent statistics is China supports this conclusion, whereby the death rate for colorectal cancer has been steadily increasing in the country, whilst the overall mortality for gastric, esophageal and cervical cancers has gradually declined (15). An approximate $10 \%$ of colorectal malignancies are attributable to germ-line mutations, while the remaining majority of these cancers are related to procarcinogenic exposures occurred years or even decades before the diagnosis (16). Therefore, the positive association between colorectal cancer and HDI, as shown in Figure 1, suggests that reinforcement or urgent establishment of effective preventive measures and accurate screening 
programs may be advisable (17), especially in countries undergoing considerable societal and economic changes, and adopting more Western lifestyles (4).

\section{Acknowledgments}

None.

\section{Footnote}

Conflicts of Interest: The authors have no conflicts of interest to declare.

Ethical Statement: The authors are accountable for all aspects of the work in ensuring that questions related to the accuracy or integrity of any part of the work are appropriately investigated and resolved.

\section{References}

1. American Cancer Society. Colorectal Cancer. Available online: https://www.cancer.org/cancer/colon-rectal-cancer. html. Last access: July 4, 2019.

2. Fleming M, Ravula S, Tatishchev SF, et al. Colorectal carcinoma: Pathologic aspects. J Gastrointest Oncol 2012;3:153-73.

3. International Agency for Research on Cancer. Cancer Fact Sheets. Available online: http://gco.iarc.fr/today/factsheets-cancers. Last access: July 4, 2019.

4. Arnold M, Sierra MS, Laversanne M, et al. Global patterns and trends in colorectal cancer incidence and mortality. Gut 2017;66:683-91.

5. Duineveld LA, van Asselt KM, Bemelman WA, et al. Symptomatic and Asymptomatic Colon Cancer Recurrence: A Multicenter Cohort Study. Ann Fam Med 2016;14:215-20.

6. World Health Association. Mortality Database. Available online: https://www.who.int/healthinfo/global_burden_ disease/estimates/en/. Last access: July 4, 2019.

Cite this article as: Mattiuzzi C, Sanchis-Gomar F, Lippi G. Concise update on colorectal cancer epidemiology. Ann Transl Med 2019;7(21):609. doi: 10.21037/atm.2019.07.91
7. Danese E, Montagnana M. Epigenetics of colorectal cancer: emerging circulating diagnostic and prognostic biomarkers. Ann Transl Med 2017;5:279.

8. Mondaca S, Yaeger R. Colorectal cancer genomics and designing rational trials. Ann Transl Med 2018;6:159.

9. Smyth E, Khan K, Valeri N. Translational research and application of basic biology to clinical trial development in GI cancers. Ann Transl Med 2018;6:164.

10. Moreno CC, Mittal PK, Sullivan PS, et al. Colorectal Cancer Initial Diagnosis: Screening Colonoscopy, Diagnostic Colonoscopy, or Emergent Surgery, and Tumor Stage and Size at Initial Presentation. Clin Colorectal Cancer 2016;15:67-73.

11. Shaukat A, Mongin SJ, Geisser MS, et al. Long-term mortality after screening for colorectal cancer. $\mathrm{N} \mathrm{Engl} \mathrm{J}$ Med 2013;369:1106-14.

12. World Cancer Research Fund/American Institute for Cancer Research. Continuous Update Project Expert Report 2018. Diet, nutrition, physical activity and colorectal cancer. Available online: https://www.wcrf.org/ dietandcancer. Last access: July 4, 2019.

13. Schwingshackl L, Schwedhelm C, Galbete C, et al. Adherence to Mediterranean Diet and Risk of Cancer: An Updated Systematic Review and Meta-Analysis. Nutrients 2017;9. doi: 10.3390/nu9101063.

14. Azeem S, Gillani SW, Siddiqui A, et al. Diet and Colorectal Cancer Risk in Asia--a Systematic Review. Asian Pac J Cancer Prev 2015;16:5389-96.

15. Bode AM, Dong Z, Wang H. Cancer prevention and control: alarming challenges in China. Natl Sci Rev 2016;3:117-27.

16. Rattray NJW, Charkoftaki G, Rattray Z, et al. Environmental influences in the etiology of colorectal cancer: the premise of metabolomics. Curr Pharmacol Rep 2017;3:114-25.

17. Montagnana M, Lippi G. Cancer diagnostics: current concepts and future perspectives. Ann Transl Med 2017;5:268. 